\title{
Influence of Quadrupole and Octupole Electron-Phonon Coupling on the Low-Field Transport Properties of n-type Silicon
}

\author{
Gram, N. O.; Jørgensen, Mogens Hoffmann
}

Published in:

Physical Review B

Link to article, DOI:

10.1103/PhysRevB.8.3902

Publication date:

1973

Document Version

Publisher's PDF, also known as Version of record

Link back to DTU Orbit

Citation (APA):

Gram, N. O., \& Jørgensen, M. H. (1973). Influence of Quadrupole and Octupole Electron-Phonon Coupling on the Low-Field Transport Properties of $\mathrm{n}$-type Silicon. Physical Review B, 8(8), 3902-3907.

https://doi.org/10.1103/PhysRevB.8.3902

\section{General rights}

Copyright and moral rights for the publications made accessible in the public portal are retained by the authors and/or other copyright owners and it is a condition of accessing publications that users recognise and abide by the legal requirements associated with these rights.

- Users may download and print one copy of any publication from the public portal for the purpose of private study or research.

- You may not further distribute the material or use it for any profit-making activity or commercial gain

- You may freely distribute the URL identifying the publication in the public portal 


\title{
Influence of Quadrupole and Octupole Electron-Phonon Coupling on the Low-Field Transport Properties of $n$-type Silicon
}

\author{
N. O. Gram and M. H. Jorgensen \\ Physics Laboratory III, Technical University of Denmark, Lyngby, Denmark \\ (Received 5 April 1973)
}

Comparison of low-field mobility and piezoresistance data with calculations gives support to the importance of quadrupole and octupole electron-phonon interactions in $\boldsymbol{n}$-type silicon.

\section{INTRODUCTION}

Several attempts in the past few years to assign a set of scattering parameters to $n$-type $\mathrm{Si}$, such that both low - and high -field phenomena could be predicted, have resulted in deformation potentials $\Xi_{u}$ and $\Xi_{d}$ which are not consistent with stress optical experiments. ${ }^{1-3}$ This paper deals with lowfield transport properties of $n$-type $\mathrm{Si}$ at $77 \mathrm{~K}$. It will be shown that it is not possible to obtain agreement with both mobility and piezoresistance measurements by taking into account only impurity scattering and interaction with acoustic and optical phonons via the conventional deformation-potential coupling. ${ }^{4}$ Agreement between theory and experiment can be obtained, however, by including a relatively strong electrical-multipole interaction. This is thought to be the first example of the practical importance of electrical-multipole interactions in electronic-transport calculations for semiconductors. Theoretical treatments of multipole interactions have been published by Tolpygo, ${ }^{5}$ Lawaetz, ${ }^{6}$ and Pindor. ${ }^{7}$

\section{SCATTERING BY ACOUSTIC PHONONS}

In a nonpolar crystal with a diamond-type lattice such as silicon, the displacement of an atom induces a disturbance in its surrounding charge distribution resulting in an electrical moment basically of quadrupole and octupole nature. Only the short-range forces due to this moment are included in the normal acoustic-deformation-potential scheme. By also taking the long-range forces into account one obtains an effective deformation-potential tensor ${ }^{6}$

$$
\Xi_{i j}=\Xi_{u}\left[\left(r_{1}+r_{2} \hat{q}_{i}^{2}\right) \delta_{i j}+\hat{\gamma}_{i} \hat{\gamma}_{j}\right]
$$

where $r_{1} \equiv\left(\Xi_{d}+C_{1}\right) / \Xi_{u}$ and $r_{2} \equiv C_{2} / \Xi_{u}$. $\quad \Xi_{u}$ and $\Xi_{d}$ are the convential deformation-potential constants, ${ }^{4} \hat{q}$ is a unit phonon wave vector, and $\hat{\gamma}$ is a unit vector parallel to the valley axis. $C_{1}$ and $C_{2}$ are related to the long-range multipole interactions. As only quadrupole and octupole interaction are of interest to the present study, one finds ${ }^{6}$

$$
C_{1}=C_{\text {qua }}-\frac{3}{5} C_{\text {oct }}
$$

and

$$
C_{2}=C_{\text {oct }}-C_{\text {qua }},
$$

$C_{\text {qua }}$ and $C_{\text {oct }}$ being quadrupole and octupole coupling coefficients. The additional $C_{3}$ term introduced by Lawaetz ${ }^{6}$ is zero due to symmetry.

Following Herring and Vogt ${ }^{4}$ we define two effective deformation-potential constants

$\Xi_{1}^{2} \equiv \Xi_{u}^{2}\left[2\left\langle\left\langle\left(\sin \theta_{1}-\sin \theta_{2} \cos \Delta \phi\right) \sin \theta_{1} \Gamma\right\rangle_{\Omega_{1}}\right\rangle_{\Omega_{2}}\right]$

and

$$
\Xi_{11}^{2} \equiv \Xi_{u}^{2}\left[4\left\langle\left\langle\left(\cos \theta_{1}-\cos \theta_{2}\right) \cos \theta_{1} \Gamma\right\rangle_{\Omega_{1}}\right\rangle_{\Omega_{2}}\right] .
$$

The angles determine the directions of the electron wave vectors before and after the collisions. Averaging over solid angles is carried out in a Herring-and-Vogt transformed space. ${ }^{4} \Gamma$ (which except for a trivial factor is equal to the scattering-matrix-element squared) can be written

$$
\Gamma=\sum_{\alpha}\left\langle u^{2}\right\rangle \frac{\sum_{i j}\left\{\left[\left(r_{1}+r_{2} \hat{q}_{i}^{2}\right) ! \delta_{i j}+\hat{\gamma}_{i} \hat{\gamma}_{j}\right] \hat{e}_{q \alpha i} \hat{q}_{j}\right\}^{2}}{u_{\alpha}^{2}},
$$

where $\alpha$ indicates summation over the three acoustic polarization directions. $\hat{e}_{\alpha \alpha}$ is a unit polarization vector, $u_{\alpha}$ is the sound velocity, and $\left\langle u^{2}\right\rangle$ $=\left(3 c_{11}+2 c_{12}+4 c_{44}\right) / 5 \rho$ is an average-sound-velocity squared introduced to make $\Gamma$ dimensionless.

In the case of longitudinally polarized phonons,

$$
u_{\alpha}^{2} \equiv u_{i}^{2}=\frac{1}{\rho}\left[c_{11}-\left(c_{11}-c_{12}-2 c_{44}\right)\left(1-\sum_{i} \hat{q}_{i}^{4}\right)\right]
$$

and in the case of transverse phonons,

$u_{\alpha}^{2} \equiv u_{t}^{2}=\frac{1}{\rho}\left(c_{44}+\left(c_{11}-c_{12}-2 c_{44}\right) \sum_{i}\left(\hat{e}_{a t i} \hat{q}_{i}\right)^{2}\right)$,

$t$ equal to $t 1$ or $t 2$ representing the two transverse modes. Evaluation of the effective deformationpotentials yields

$$
\begin{aligned}
\Xi_{1}^{2}=\Xi_{u}^{2}\left(K_{\mathrm{TO}}+K_{T 1} r_{1}+K_{T 2} r_{1}^{2}+L_{T 1} r_{2}\right. \\
\\
\left.+L_{T 2} r_{2}^{2}+M_{T 2} r_{1} r_{2}\right) \\
\Xi_{I 1}^{2}=\Xi_{u}^{2}\left(K_{L O}+K_{L 1} r_{1}+K_{L 2} r_{1}^{2}+L_{L 1} r_{2}\right. \\
\left.+L_{L 2} r_{2}^{2}+M_{L 2} r_{1} r_{2}\right),
\end{aligned}
$$


TABLE I. Expansion coefficients for the effective deformation-potential constants. Results using three different procedures are shown: (i) the Herring and Vogt approximation (Ref. 4); (ii) assumed isotropic sound velocity; and (iii) a detailed procedure where the elastic anisotropy has been taken fully into account and the correct phonon polarization vectors have been used.

\begin{tabular}{lccc}
\hline & Herring and Vogt & Isotropic sound velocity & Detailed theory \\
\hline$K_{\mathrm{TO}} ; K_{\mathrm{LO}}$ & $1.07 ; 1.62$ & $0.98 ; 1.39$ & $1.04 ; 1.58$ \\
$K_{T 1} ; K_{L 1}$ & $1.15 ; 2.40$ & $1.17 ; 2.18$ & $1.14 ; 2.46$ \\
$K_{T 2} ; K_{L 2}$ & $1.33 ; 1.40$ & $1.35 ; 1.34$ & $1.34 ; 1.40$ \\
$L_{T 1} ; L_{T 1}$ & $\ldots$ & $\ldots$ & $0.85 ; 2.48$ \\
$L_{T 2} ; L_{L 2}$ & $\ldots$ & $\ldots$ & $0.73 ; 1.10$ \\
$M_{T 2} ; M_{L 2}$ & $\ldots$ & $\ldots$ & $1.70 ; 2.25$ \\
\hline \hline
\end{tabular}

where the coefficients $K, L$, and $M$ depend only on the effective-mass ratio and the elastic constants.

Table I shows the $K$ coefficients as calculated using three different procedures. In the Herring and Vogt approximation, the integration is carried out by first calculating $\Gamma$ for the main symmetry directions and then interpolating with Legendre polynomials. In the other approximation with

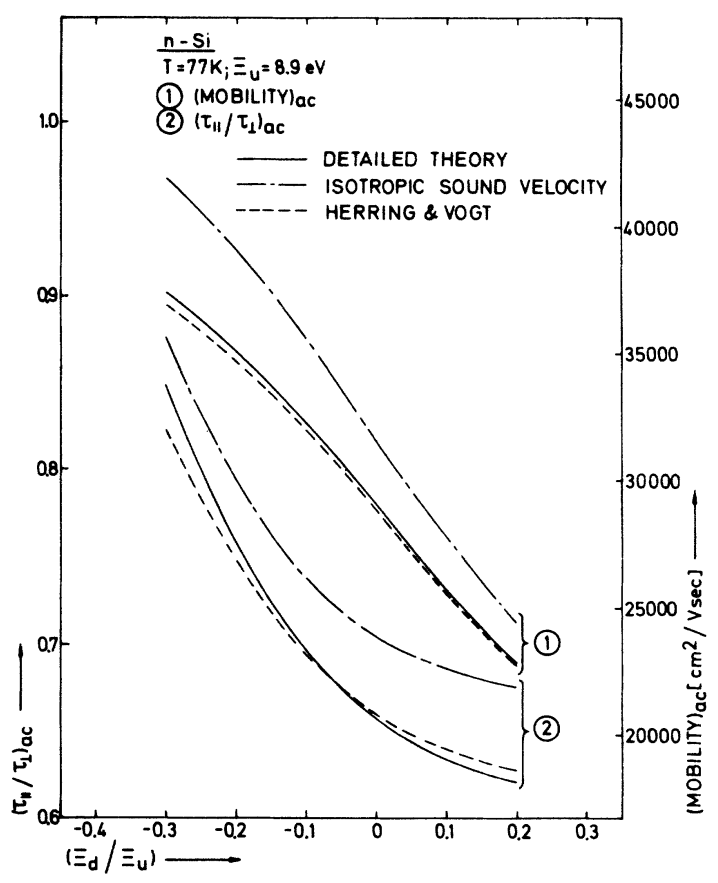

FIG. 1. Calculated anisotropy factor $\left(\tau_{11} / \tau_{\perp}\right)_{\mathrm{ac}}$ and acoustic-phonon-limited low-field mobility as a function of the ratio of acoustic deformation potentials $\Xi_{d} / \Xi_{u}$. Results using three different procedures are shown: (i) Herring and Vogt approximation (dotted curves) (Ref. 4); (ii) assumed isotropic sound velocity (dash-dotted curves); (iii) a detailed procedure where the elastic anisotropy has been taken fully into account and the correct phonon polarization vectors have been used (full line curves). isotropic sound velocity, the anisotropic elastic constants have been replaced by average values obtained by averaging over the $\langle 100\rangle,\langle 110\rangle$, and $\langle 111\rangle$ crystal directions. A longitudinal elastic constant equal to $\frac{1}{39}\left(22 c_{11}+17 c_{12}+34 c_{44}\right)$ and a transverse constant equal to $\frac{1}{78}\left(17 c_{11}+17 c_{12}+44 c_{44}\right)$ are found. This approximation leads to coefficients very similar to those used by Samoilovich et al. ${ }^{8}$ The third column in Table I gives the results from a more detailed procedure. In this we have taken the elastic anisotropy fully into account. Also, correct phonon polarization vectors have been used. To our knowledge results of such a detailed calculation have not previously been reported.

The approximative methods result in coefficients deviating up to $8 \%$ from the exact coefficients. For the range of deformation potentials of interest for the present study, these discrepancies may cause deviations up to $10 \%$ in the acoustic-phononlimited low-field mobility (Fig. 1):

$$
\mu_{\mathrm{ac}} \propto\left(2 / \Xi_{1}^{2}+\left(m_{1} / m_{11}\right) / \Xi_{11}^{2}\right) \text {. }
$$

Similarly, the anisotropy factor defined as the ratio of longitudinal to transverse relaxation time,

$$
\left(\tau_{11} / \tau_{\perp}\right)_{\mathrm{ac}}=\Xi_{\perp}^{2} / \Xi_{\| 1}^{2} \text {, }
$$

may deviate up to $10 \%$ from the detailed theory (Fig. 1). In the following calculations we have therefore used the detailed procedure. The $L$ and $M$ coefficients related to multipole electron-phonon interactions are shown in Table $I$.

\section{CALCULATIONS WITHOUT LONG-RANGE INTERACTIONS}

A preliminary set of scattering parameters ${ }^{9}$ determined by fitting to the low-field mobility between 77 and $300 \mathrm{~K}$ and to the drift velocity at $77 \mathrm{~K}$ as a function of field in the $\langle 111\rangle$ and $\langle 100\rangle$ directions is shown in Table II. The fitting procedure is especially sensitive for the $\langle 100\rangle$ velocity-field characteristic which has a characteristic "kink" structure. ${ }^{10-12}$ Scattering by impurities and acoustic and intervalley phonons (of $f$ and $g$ type) have been 


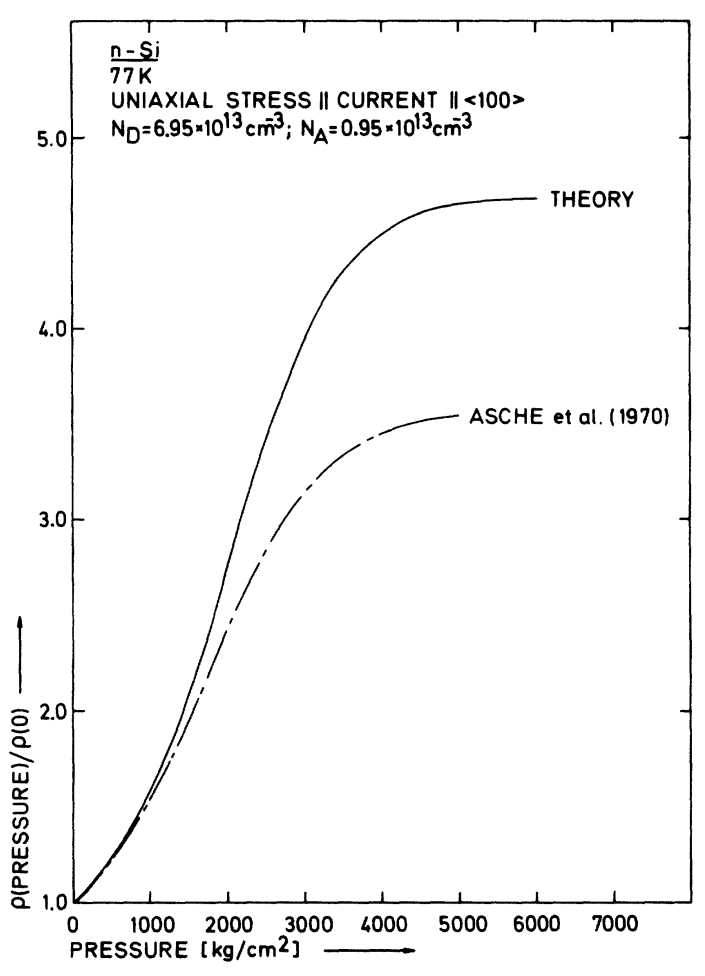

FIG. 2. Comparison between calculated and measured piezoresistance values at $77 \mathrm{~K}$. Current and uniaxial stress parallel to a $\langle 100\rangle$ crystal direction. The theoretical values have been obtained using the preliminary set of scattering parameters shown in Table II.

TABLE II. Preliminary (Ref. 9) and final scattering parameters.

\begin{tabular}{lcc}
\hline & Preliminary & Final \\
\hline$\Xi_{u}$ & $8.9 \mathrm{eV}$ & $8.6 \mathrm{eV}$ \\
$\Xi_{d}$ & $1 \mathrm{eV}$ & $0.1 \mathrm{eV}$ \\
$C_{\text {qua }}$ & $\cdots$ & $2.7 \mathrm{eV}$ \\
$C_{\text {oct }}$ & $\cdots$ & $-2.1 \mathrm{eV}$ \\
$D_{g^{2}}$ & $1.0 \times 10^{10} \mathrm{eV} / \mathrm{m}$ & $1.0 \times 10^{10} \mathrm{eV} / \mathrm{m}$ \\
$\hbar \omega_{g^{2}}(\mathrm{LO})^{2}$ & $62 \mathrm{meV}$ & $62 \mathrm{meV}$ \\
$D_{g^{1}}$ & $0.18 \times 10^{10} \mathrm{eV} / \mathrm{m}$ & $0.18 \times 10^{10} \mathrm{eV} / \mathrm{m}$ \\
$\hbar \omega_{g 1}(\mathrm{LA})^{2}$ & $25 \mathrm{meV}$ & $25 \mathrm{meV}$ \\
$D_{f 2}$ & $6.2 \times 10^{10} \mathrm{eV} / \mathrm{m}$ & $6.2 \times 10^{10} \mathrm{eV} / \mathrm{m}$ \\
$\hbar \omega_{f 2}(\mathrm{LA})^{\mathrm{b}}$ & $48 \mathrm{meV}$ & $48 \mathrm{meV}$ \\
$D_{f 1}$ & $0.18 \times 10^{10} \mathrm{eV} / \mathrm{m}$ & $0.18 \times 10^{10} \mathrm{eV} / \mathrm{m}$ \\
$\hbar \omega_{f 1}(\mathrm{TA})^{\mathrm{b}}$ & $23 \mathrm{meV}$ & $23 \mathrm{meV}$ \\
\hline \hline
\end{tabular}

${ }^{a}$ Phonon coupling with parallel valley on the same axis in $k$ space.

${ }^{b}$ Phonon coupling with one out of four perpendicular valleys.

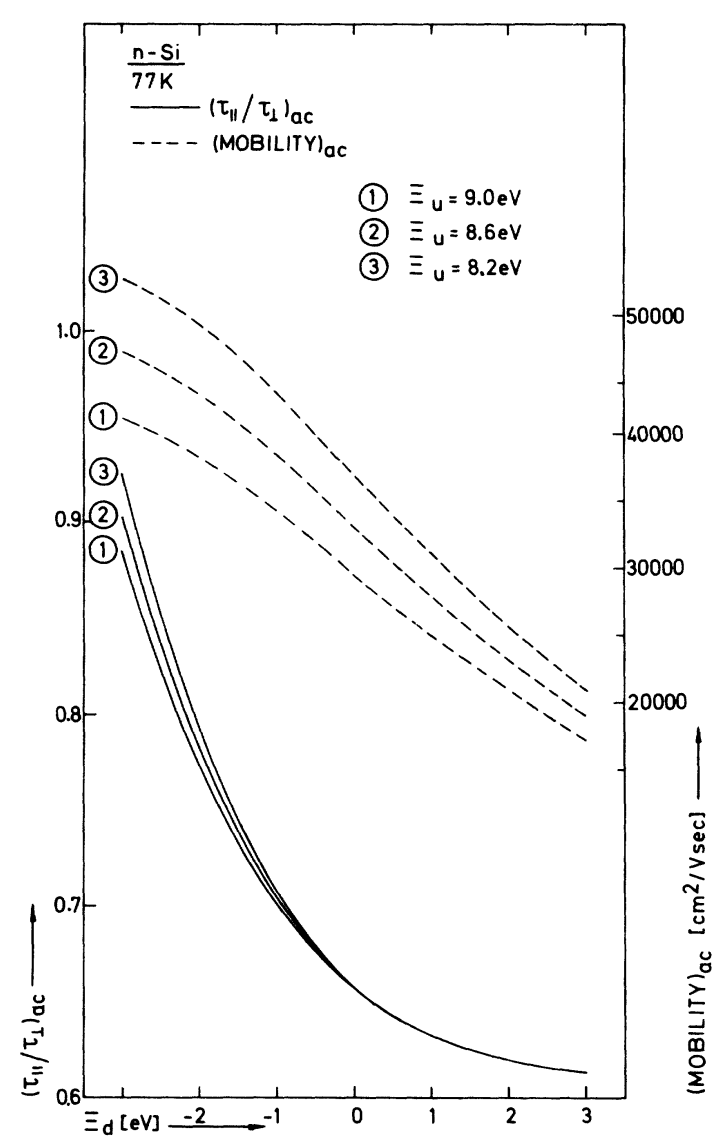

FIG. 3. Anisotropy factor $\left(\tau_{11} / \tau_{\perp}\right)_{\text {ac }}$ (full line curves) and acoustic-phonon-limited low-field mobility (dotted curves) vs the acoustic deformation potential $\Xi_{d}$ with the acoustic deformation potential $\Xi_{u}$ as parameter. Longrange multipole interactions have not been taken into account.

included. ${ }^{13}$ Here inclusion of anisotropic impurity scattering is essential as it substantially reduces the total anisotropy. Both impurity and intervalley phonon scattering are treated by standard procedures. ${ }^{10}$ Multipole interactions have not yet been taken into account, i. e., $C_{1}=C_{2}=0 \mathrm{eV}$.

It is important to notice that in order to reproduce the experimental results mentioned above one has to include a weak coupling to two low-energy intervalley phonons. Both processes are forbidden to zeroth order by symmetry. ${ }^{14}$ Furthermore, the required acoustic deformation potential $\Xi_{u}=8.9 \mathrm{eV}$ is $4 \%$ higher than the average value determined by Balslev. ${ }^{3}$

More serious, however, is the fact that the calculated and measured piezoresistance values at $77 \mathrm{~K}$ for stress and current along a $\langle 100\rangle$ direction deviate considerably (Fig. 2). The theoretical variation is too strong for the parameters used, 


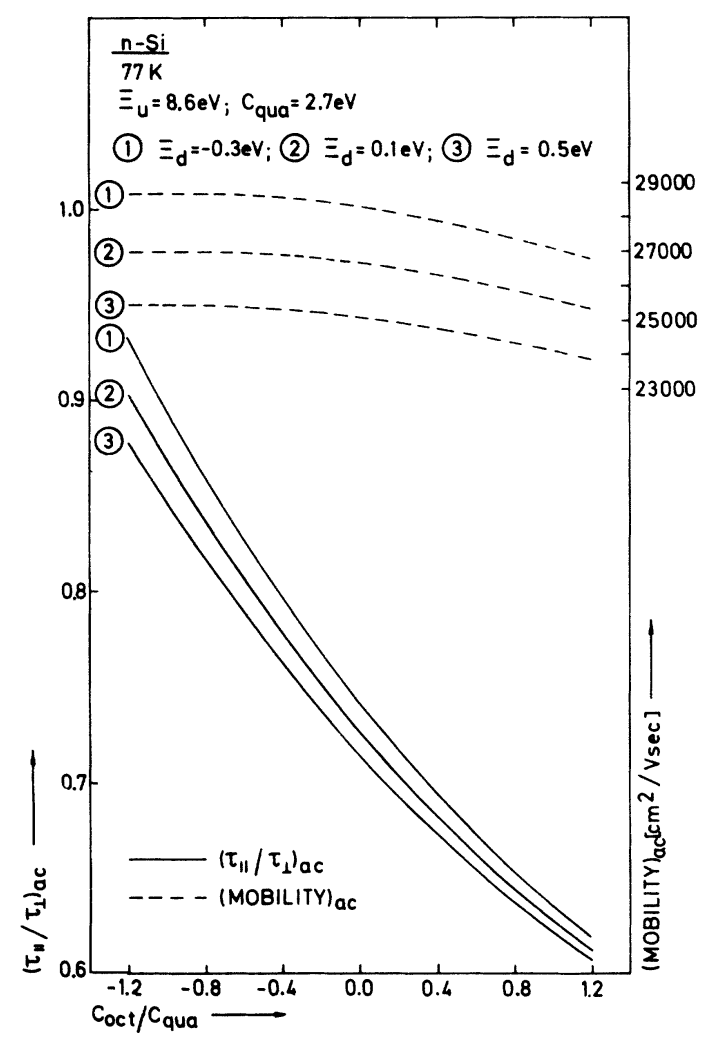

FIG. 4. Acoustic-phonon-limited low-field mobility (dotted curves) and anisotropy factor (full line curves) vs the ratio of octupole to quadrupole coupling constants. $C_{\text {qua }}=2.7 \mathrm{eV}$ (Ref. 7). $\Xi_{u}=8.6 \mathrm{eV}$ (Ref. 3). The acoustic deformation potential $\Xi_{d}$ is used as parameter.

meaning that the assumed anisotropy is too large $\left(\tau_{11} / \tau_{\perp}\right.$ too far from unity).

The question is now whether it is possible by taking only conventional phonon scattering into account to bring $\tau_{11} / \tau_{1}$ closer to unity while at the same time keeping the phonon-limited mobility fixed at the experimental value of $27000 \mathrm{~cm}^{2}$ / V sec. ${ }^{13}$ At this temperature, intervalley phonon scattering in $n$-type $\mathrm{Si}$ plays an insignificant role at low electric fields. By neglecting it completely we only increase the mobility by $2 \%$ and the total anisotropy by $0.2 \%$. We are thus left with the possibility of changing the acoustic deformation potentials to obtain-if possible - the desired anisotropy factor and mobility. Figure 3 shows $\left(\tau_{11} / \tau_{\perp}\right)_{\text {ac }}$ and $\mu_{\mathrm{ac}}$ versus the deformation potential $\Xi_{d}$ with $\Xi_{u}$ as parameter. Our preliminary set of parameters leads to $\left(\tau_{11} / \tau_{\perp}\right)_{\mathrm{ac}}=0.63$, whereas Asche et al. ${ }^{1}$ have deduced a value of 0.81 from piezoresistance measurements. It is possible to change $\left(\tau_{\|} / \tau_{1}\right)_{\mathrm{ac}}$ from 0.63 to 0.81 by decreasing $\Xi_{d}$. At the same time, however, the mobility increases by as much as $50 \%$. Therefore this procedure does not lead to a new set of scattering parameters consistent with the experimental data.

\section{CALCULATIONS INCLUDING LONG-RANGE INTERACTIONS}

In this section quadrupole and octupole interaction are taken into account. In Fig. 4 the acousticlimited low-field mobility and the anisotropy factor are shown versus the ratio of octupole to quadrupole coupling constants. $C_{\text {qua }}$ is taken to be $2.7 \mathrm{eV}$ as calculated by Pindor. ${ }^{7} \Xi_{u}=8.6 \mathrm{eV}$ as measured by Balslev. ${ }^{3} \Xi_{d}$ is used as parameter.

Contrary to the results presented above, a 50\% increase in $\left(\tau_{11} / \tau_{1}\right)_{\text {ac }}$ only results in less than $4 \%$ increase in the phonon-limited mobility. It is thus possible to determine a new set of scattering pa-

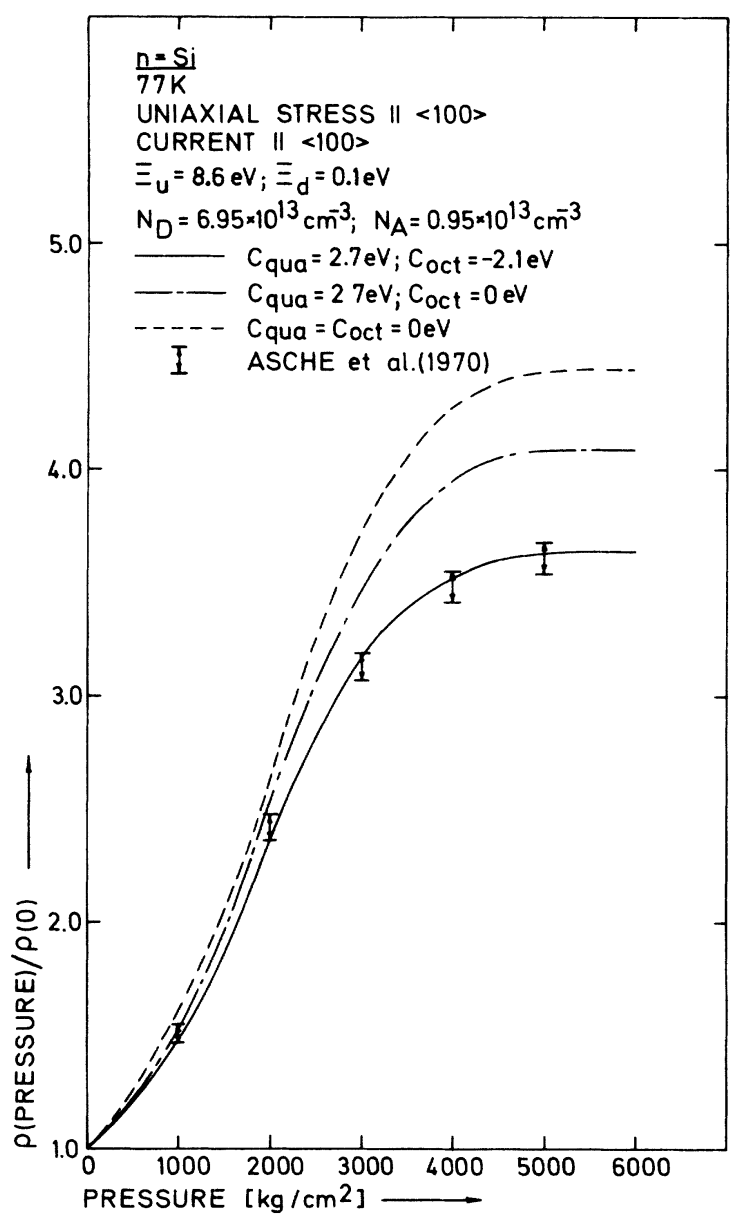

FIG. 5. Comparison between calculated piezoresistance values (full line curve) and measured (Ref. 1) values (vertical bars) of resistivity vs pressure for stress and current parallel to a $\langle 100\rangle$ crystal direction. The theoretical values have been obtained using the final set of scattering parameters shown in Table II. Calculated results without octupole coupling (dash-dotted curve) and with no multipole coupling (dotted curve) are also shown. 


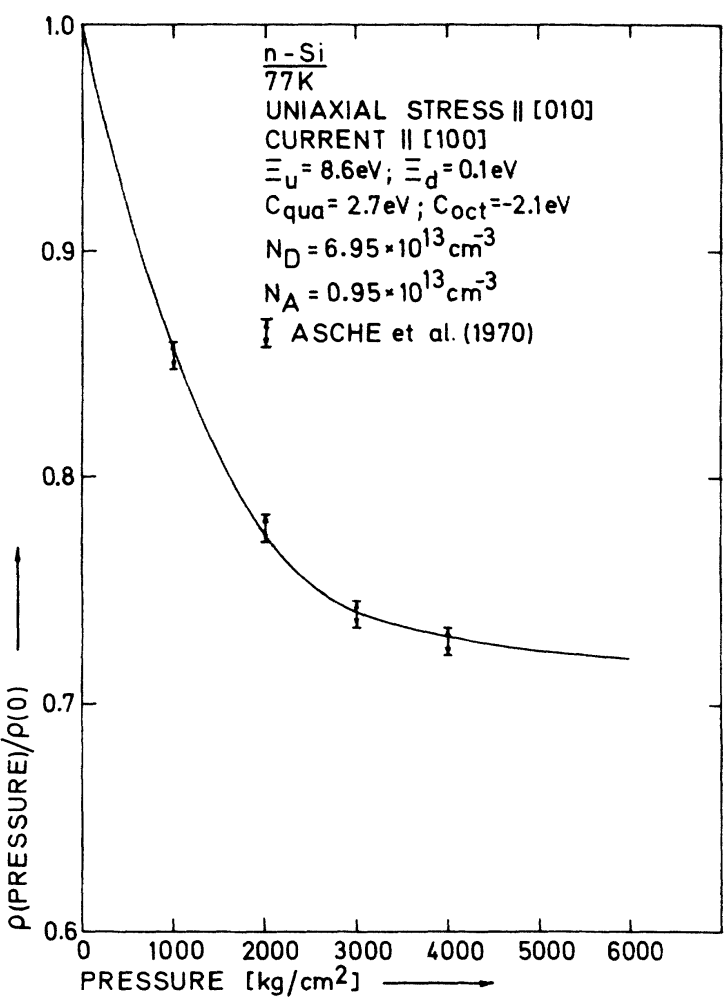

FIG. 6. Comparison between calculated piezoresistance values (full line curve) and measured values by Asche et al. (Ref. 1) (vertical bars). Current along the [100] and uniaxial stress along the [010] direction. The theoretical results have been obtained using the final set of scattering parameters shown in Table II.

rameters -including quadrupole and octupole electron-phonon interaction-which reproduces the piezoresistance measurements by Asche et al. ${ }^{1}$ Agreement is obtained by taking $\Xi_{d}=0.1 \mathrm{eV}$ and the octupole coupling constant equal to $-2.1 \mathrm{eV}$ (Table II). This final set of scattering parameters leads to $\tau_{11} / \tau_{\perp}=0.83$ as compared to 0.81 found by Asche et al. ${ }^{1}$ The calculated results are shown as the full line curve and the experimental results as vertical bars in Fig. 5. In the same figure is also shown the resistivity versus pressure for $C_{\text {oct }}=0$ $\mathrm{eV}$ (dash-dotted curve) and for no multipole interaction $C_{\text {qua }}=C_{\text {oct }}=0 \mathrm{eV}$ (dotted curve). Both situations lead to anisotropies which are too large.

An independent test of the final set of scattering parameters can be obtained by comparing calculated and measured resistivity variation for a new experimental configuration. This has been done in Fig. 6 where results for current along the [110] and uniaxial stress along the [010] direction are presented. The agreement between theoretical and experimental values is as good as in the case of the experimental configuration considered for the fitting procedure.

\section{CONCLUSION}

The results indicate that the normally neglected long-range quadrupole and octupole electron-phonon interaction may be quite important in the case of silicon. It has been demonstrated that it is not possible to obtain agreement with experimental results by taking into account only impurity scattering and interaction with acoustic and intervalley phonons via the conventional deformation-potential coupling. However, by including quadrupole and octupole electron-phonon interactions, correct values for both low-field mobility and the piezoresistance effects have been obtained. The deformation-potential constant $\Xi_{u}$ is deduced from piezooptical measurements, ${ }^{3}$ whereas the quadrupole coupling constant is set equal to the theoretically determined value. ${ }^{7}$ The value of the fitting parameters $\Xi_{d}=0.1 \mathrm{eV}$ and $C_{\text {oct }}=-2.1 \mathrm{eV}$ are in agreement with preliminary theoretical considerations by Lawaetz. ${ }^{15} \Xi_{d}$ should lie between 0 and $1 \mathrm{eV}$ and the numerical value of the octupole coupling constant should be of the same order of magnitude as the quadrupole coupling constant.

During the investigation it has also been demonstrated that the normal approximations applied in connection with low -field mobility calculations (in particular the assumption of isotropic sound velocity) lead to mobilities which may deviate up to $10 \%$ from the exact values.

As we have confined our considerations to a lattice temperature of $77 \mathrm{~K}$, no further information concerning the two forbidden low-energy intervalley phonons can be deduced. However, hot-electron calculations including multipole interaction are in progress and during this investigation the need for including the forbidden phonons will be examined.

\section{ACKNOWLEDGMENTS}

Thanks are due Dr. P. Lawaetz and Dr. N. I. Meyer for valuable discussions.
${ }^{1}$ M. Asche, V. M. Vasetsky, A. G. Maximchuk, and O. G. Sarbey, Ukr. Fiz. Zh. 15, 1692 (1970).

${ }^{2}$ D. Günther, dissertation (Humboldt University, 1970) (unpublished).

${ }^{3}$ I. Balslev, Phys. Rev. 143, 636 (1966).

${ }^{4}$ C. Herring and E. Vogt, Phys. Rev. 101, 944 (1956).

${ }^{s}$ K. B. Tolpygo, Fiz. Tverd. Tela 4, 1765 (1962) [Sov.
Phys.-Solid State 4, 1297 (1963)]

${ }^{6}$ P. Lawaetz, Phys. Rev. 183, 730 (1969).

${ }^{7}$ A. J. Pindor, J. Phys. C 5, 2357 (1972).

${ }^{8}$ A. G. Samoilovich, J. Ya. Korenblit, J. V. Dakhovskii, and V. D. Iskara, Fiz. Tverd. Tela 3, 3285 (1961) [Sov. Phys.-Solid State 3, 2385 (1962)].

${ }^{9}$ M. H. Jorgensen, N. O. Gram, and N. I. Meyer, Solid State 
Commun. 10, 337 (1972).

${ }^{10}$ M. H. Jørgensen, Phys. Rev. 156, 834 (1967).

${ }^{11} M$. H. Jørgensen, N. I. Meyer, and K. J. Schmidt-Tiedmann, in Proceedings of the Seventh International Conference on the Physics of Semiconductors (Academic, New York, 1964), p. 457.
${ }^{12}$ M. Asche, B. L. Boichenko, and O. G. Sarbey, Phys. Status Solidi 9, 323 (1965).

${ }^{13}$ D. Long, Phys. Rev. 120, 2024 (1960).

${ }^{14}$ H. W. Streitwolf, Phys. Status Solidi 37, K47 (1970).

${ }^{15} \mathrm{P}$. Lawaetz (private communication). 